\title{
NOUVELle
}

\section{L'expression des récepteurs de chimiokines à la surface des lymphocytes $T$ reflète la dissémination métastatique du mélanome}

Nicolas Jacquelot ${ }^{1}$, David P. Enot ${ }^{2}$, Caroline Flament ${ }^{1}$, Stéphane Dalle ${ }^{3}$, Laurence Zitvogel ${ }^{1}$

\author{
${ }^{1}$ Inserm U1015, Gustave Roussy Cancer Campus, \\ 114, rue Edouard Vaillant, 94800 Villejuif, France ; \\ 2 Metabolomics and cell biology platforms, \\ Gustave Roussy Cancer Campus, 94800 Villejuif, France ; \\ ${ }^{3}$ Centre Hospitalier Lyon Sud, Cancer Research Center of Lyon \\ et Université Claude Bernard Lyon 1, Lyon, France. \\ laurence.zitvogel@orange.fr \\ jacquelot.nicolas@gmail.com
}

> La migration cellulaire au sein de l'organisme s'effectue grâce à la coordination et à l'intégration d'un ensemble de signaux complexes faisant principalement intervenir les couples chimiokines/récepteurs de chimiokines. Ces récepteurs de chimiokines sont des protéines membranaires, composées de 7 domaines transmembranaires, couplées aux protéines G. Leur expression est indispensable pour la mise en place et le bon fonctionnement du système immunitaire et participe au maintien de notre homéostasie. Lors d'un processus inflammatoire, notamment tumoral, les cellules immunes doivent exprimer un ensemble de récepteurs de chimiokines adéquats, guidant alors la migration des cellules dans les tissus cibles grâce à un gradient chimiotactique [1]. L'étude de ces récepteurs dans les pathologies tumorales a permis d'améliorer la compréhension de nombre de mécanismes impliqués. Nous savons que l'infiltrat immunitaire ${ }^{1}$ au sein des lésions tumorales est décisif pour le devenir clinique des patients $[2,13]$

$(\rightarrow)$.

Néanmoins, dans le mélanome, connu pour exprimer de nombreux récepteurs de chimiokines

\section{$(\rightarrow)$ Voir le numéro} thématique Microenvironnements tumoraux : conflictuels et complémentaires, $m / s n^{\circ} 4$, avril 2014 participant activement à la dissémination de la maladie [3], seuls quelques

${ }^{1}$ Ensemble des cellules immunitaires présentes sur le site de la tumeur. études non conclusives se sont focalisées sur l'analyse globale de ces récepteurs à la surface des lymphocytes T [4]. Pourtant, l'expression de ces molécules sur ces populations immunitaires pourrait permettre de discerner l'historique de leur migration entre tumeur et périphérie, pouvant ainsi améliorer la prise en charge diagnostique et pronostique de ces patients.

Dans ce but, nous avons exploré l'expression de neuf de ces récepteurs de chimiokines à la surface des lymphocytes T circulants et intratumoraux chez des patients atteints de mélanome. Ces récepteurs sont impliqués dans différentes voies de migration, notamment vers la peau (CCR[chemokine ( $C$-C motif) receptor] 10 et CLA [cutaneous lymphocyte antigen]), les organes lymphoïdes secondaires (CXCR[chemokine ( $C-X-C$ motif) receptor]5), le tube digestif (CCR9 et $\mathrm{CD} 103^{2}$ ) ou la moelle osseuse, le cerveau et les poumons (CXCR4), mais également dans des processus de différenciation cellulaire (CRTH2 aussi appelé prostaglandin D2 receptor 2, CXCR3, CCR6 et CCR10) [1, 3]. La discrimination des différentes sous-populations de lymphocytes $T$ sur la base de l'expression des molécules CCR7 et CD45RA nous a permis de montrer que, premièrement, il existe une expression similaire de ces récepteurs de chimiokines sur les lymphocytes $\mathrm{T} C D 4^{+}$et $\mathrm{T} \mathrm{CD} 8^{+}$, sauf exception

2 CD103 est une intégrine, récepteur pour la $\varepsilon$-cadhérine épithéliale. pour CXCR4 et CD103, et secondairement, que ce schéma d'expression est comparable entre les lymphocytes $T$ naifs $\left(C C R 7^{+} C D 45 R A^{+}\right)$, centraux mémoires $\left(C C R 7^{+} C D 45 R A^{-}\right)$, effecteurs mémoires (CCR7 ${ }^{-}$CD45RA ${ }^{-}$) et terminaux effecteurs $\left(C C R 7^{-}{ }^{-C D 45 R A^{+}}\right)$[5]. Ces résultats vont donc dans le sens d'une modification générale de l'expression de ces récepteurs à la surface des lymphocytes T liée au statut de l'hôte, et notamment à la maladie.

Nous avons par ailleurs trouvé certaines signatures associant l'expression de ces récepteurs aux localisations tumorales. $\varepsilon n$ effet, la classification des patients suivant leurs localisations métastatiques (peau et ganglions, poumons, multi-métastatiques avec ou sans localisations pulmonaires) a permis de mettre en évidence 62 récepteurs de chimiokines dont le profil d'expression était significativement différent selon les localisations tumorales après normalisation avec les pourcentages obtenus chez des volontaires sains. Une perte globale d'expression, sur les lymphocytes T circulants, de CCR6 et de CXCR3 est associée préférentiellement aux localisations métastatiques cutanées et ganglionnaires. Lors d'une progression pulmonaire, nous avons observé une diminution d'expression de CXCR4, CXCR5 et CCR9. La progression vers une dissémination multi-organes est associée au contraire à une augmentation de CCR10, CD103 et de CRTH2 (Figure 1). Au sein des métastases gan- 


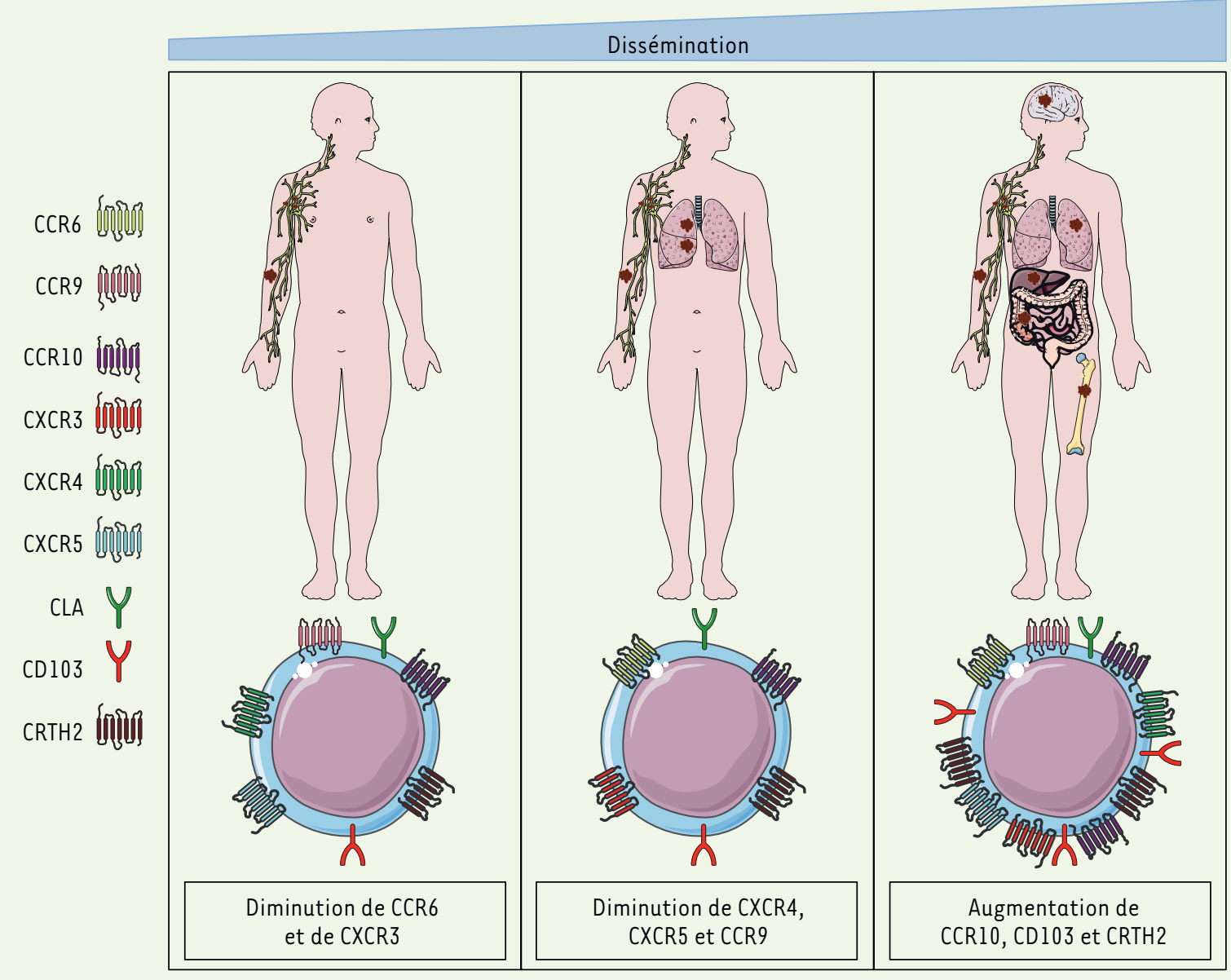

Figure 1. Illustration de la modification de l'expression des récepteurs de chimiokines lors de la dissémination du mélanome. CCR : chemokine (C-C motif) receptor; CXCR : chemokine (C-X-C motif) receptor; CLA : cutaneous lymphocyte antigen ; CD103 : cluster of differentiation 103 ; CRTH2 : prostaglandin D2 receptor 2 .

glionnaires, nous avons constaté une forte expression de CXCR3 à la surface des lymphocytes $T$, un récepteur de chimiokines largement décrit dans la littérature car considéré comme facteur de bon pronostic $[5,6]$. Nous avons également observé une augmentation de CD103 et une diminution de CCR6, CCR10, CLA et CXCR5 dans ces métastases [5].

De façon surprenante, l'expression importante de CCR9 sur les lymphocytes $T C D 8^{+}$naïfs est associée à une meilleure survie. Afin d'approfondir les mécanismes impliqués dans ce phénomène, nous avons utilisé un modèle murin de tumeurs de mélanome générées spontanément par la surexpression du proto oncogène RET dans les mélanocytes [7]. Nous avons montré un bénéfice de l'accumulation de ces lymphocytes $\mathrm{T} \mathrm{CD}^{+}$au sein de ces tumeurs [5]. En effet, nous avons trouvé une corrélation inverse entre la taille tumorale et l'infiltration de la tumeur par ces cellules $T$ $\mathrm{CD}^{+} \mathrm{CCR}^{+}$naïves. De plus, le blocage par un anticorps anti-CCL(chemokine [C-C motif] ligand)25, ligand du récepteur CCR9, favorise la croissance tumorale naturelle dans un modèle de sarcome. Parallèlement, le blocage de I'axe CXCR3 avec un anticorps spécifique du récepteur entraîne de façon attendue, une accélération de la croissance tumorale naturelle. L'exploration des populations immunitaires dans ces tumeurs et dans les ganglions drainants a révélé une diminution d'infiltrat des lymphocytes T CD $4^{+}$et T CD $8^{+}$lors du blocage respectivement par les anticorps anti-CCL25 et anti-CXCR3. Ces résultats montrent l'importance de cette voie CCR9/CCL25 dans l'immunosurveillance naturelle, différente de celle impliquant CXCR3 et ses ligands, et illustre donc la régulation qui existe entre le tube digestif et l'immunité systémique [8].

Récemment, la prise en charge thérapeutique du mélanome a été bouleversée. $\varepsilon n$ effet, le développement et l'utilisation d'anticorps monoclonaux ciblant les points de contrôle négatifs du système immunitaire a permis d'améliorer considérablement les réponses cliniques des 
patients atteints de mélanomes [9-11]

$(\rightarrow)$.

$(\rightarrow)$ Voir la Synthèse de $\varepsilon$. Tartour et al., $\mathrm{m} / \mathrm{s} \mathrm{n}^{\circ} 10$ octobre 2011, page 833

Malheureusement

l'utilisation de ces traitements entraîne l'apparition d'effets secondaires pouvant être graves, notamment à la suite de l'injection d'anti-CTLA-4 (cytotoxic T-lymphocyte-associated protein 4) [12]. Il est donc nécessaire d'améliorer cette balance bénéfice-risque en identifiant des biomarqueurs d'efficacité et de toxicité. Nous avons donc également étudié l'expression de ces récepteurs de chimiokines sur les lymphocytes $T$ circulants avant et pendant le traitement par anti-CTLA-4. Nous avons montré que l'augmentation en périphérie, dans la circulation, des effecteurs mémoires $\mathrm{T} \mathrm{CD8}^{+}$exprimant la glycoprotéine CLA au cours du traitement, 3 semaines après la première injection d'anti-CTLA-4, prédit un contrôle de la maladie à trois mois, avec des patients stables et des patients montrant une réponse partielle ou complète au traitement [5].

Ces résultats permettent d'ouvrir la voie vers l'étude de l'expression de ces récepteurs de chimiokines à la surface des lymphocytes $T$ comme agents diagnostiques dans le mélanome. Par ailleurs, l'investigation de l'expression de ces molécules au cours des nouvelles thérapies ciblées et immunothérapies pourraient permettre de guider plus précisément l'utilisation de ces médicaments afin d'accroître les bénéfices tout en réduisant les risques chez les patients. $\diamond$

Chemokine receptors expression

on T cells: contribution

to diagnosis of melanoma

metastasis dissemination status

\section{REMERCIEMENTS}

Nous remercions toutes les personnes ayant contribué à l'obtention de ces résultats. Les illustrations ont été réalisées à partir de Servier Medical Art. Nicolas Jacquelot a bénéficié d'une bourse du Cancéropôle Île-de-France.

\section{LIENS D'INTÉRÊT}

Les auteurs déclarent n'avoir aucun lien d'intérêt concernant les données publiées dans cet article.

\section{RÉFÉRENCES}

1. Griffith JW, Sokol CL, Luster AD. Chemokines and chemokine receptors: positioning cells for host defense and immunity. Annu Rev Immunol 2014 ; 32 : 659-702.
2. Jacquelot N, Roberti MP, Enot DP, et al. Immunophenotyping of stage III melanoma reveals parameters associated with patient prognosis. J Invest Dermatol 2016 ; 136 : 994-1001.

3. Zlotnik A, Burkhardt AM, Homey B. Homeostatic chemokine receptors and organ-specific metastasis. Nat Rev Immunol 2011; 11 : 597-606.

4. Salerno EP, Olson WC, McSkimming C, et al. T cells in the human metastatic melanoma microenvironment express site-specific homing receptors and retention integrins. Int J Cancer $2014 ; 134:$ 563-74.

5. Jacquelot N, Enot DP, Flament C, et al. Chemokine receptor patterns in lymphocytes mirror metastatic spreading in melanoma. J Clin Invest 2016; 126 : 921-37.

6. Mullins IM, Slingluff CL, Lee JK, et al. CXC chemokine receptor 3 expression by activated CD8+ T cells is associated with survival in melanoma patients with stage III disease. Cancer Res 2004 ; 64 : 7697-701.

7. Kato M, Takahashi M, Akhand AA, et al. Transgenic mouse model for skin malignant melanoma. Oncogene $1998 ; 17: 1885-8$.

8. Perez-Chanona $\varepsilon$, Trinchieri G. The role of microbiota in cancer therapy. Curr Opin Immunol 2016 ; 39 : 75-81.

9. Hodi FS, O'Day SJ, McDermott DF, et al. Improved survival with ipilimumab in patients with metastatic melanoma. N Engl J Med 2010 ; 363 : 711-23.

10. Robert C, Thomas L, Bondarenko I, et al. Ipilimumab plus dacarbazine for previously untreated metastatic melanoma. $N$ Engl J Med 2011 ; 364 : 2517-26.

11. Tartour $\varepsilon$, Sandoval F, Bonnefoy JY, Fridman WH. Immunothérapie des cancers : Succès récents et perspectives. Med Sci (Paris) 2011 ; $27: 833-41$.

12. Fecher LA, Agarwala SS, Hodi FS, Weber JS. Ipilimumab and its toxicities: a multidisciplinary approach. Oncologist $2013 ; 18: 733-43$.

13. Teillaud JL. Microenvironnements tumoraux : conflictuels et complémentaires. Med Sci (Paris) $2014 ; 30: 343-462$.

\section{NOUVELLE}

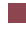 \\ Addiction par stimulation des neurones dopaminergiques mésolimbiques}

Dopamine : nécessaire ou suffisante pour induire l'addiction?

Les substances addictives ont en commun la propriété d'augmenter la concentration extracellulaire en dopamine (DA) dans le noyau accumbens (NAC), une structure cérébrale faisant partie du système de la récompense et jouant un rôle important pour la motivation et le renforcement de comportements bénéfiques à la survie. Les cibles moléculaires des substances addictives sont diverses : transporteurs des monoamines ${ }^{1}$, récepteurs aux opiacés ou aux cannabinoïdes, canaux nicotiniques ${ }^{2}$ [15] $(\rightarrow)$.

\section{$\rightarrow$ Voir la Synthèse de J. Kaufling et al., $m / s n^{\circ} 6-7$, juin-juillet 2016, page 619}

\footnotetext{
${ }^{1}$ Famille de neurotransmetteurs dérivés d'acide aminés, comprenant notamment la dopamine, la noradrénaline et la sérotonine.

${ }^{2}$ Récepteur de l'acétylcholine perméable au sodium et au potassium.
}

\author{
${ }^{1}$ Department of basic neurosciences, \\ medical faculty, university of Geneva, CMU, \\ 1 rue Michel Servet, $\mathrm{CH}-1211$ Geneva, Suisse ; \\ ${ }^{2}$ Clinic of neurology, department of clinical \\ neurosciences, Geneva university hospital, \\ CH-1211 Geneva, Suisse. \\ vincent.pascoli@unige.ch
}

Ces substances ont donc des modes d'action différents permettant d'augmenter la concentration en dopamine : soit par inhibition de sa recapture, soit par activation directe des neurones dopaminergiques, soit par inhibition d'interneurones inhibiteurs des neurones à DA.

L'augmentation de DA est-elle suffisante pour induire les effets moléculaires, cellulaires et comportementaux 